\title{
An Eco-Friendly Proposal by Integrating Chromatographic Fingerprinting and Multivariate Control Chart in a Non-Target Analysis to Evaluate Grape Juices from Different Farming Practices
}

\author{
Leonardo Valderrama, ${ }^{a}$ Bogdan Demczuk Jr., ${ }^{b}$ Patrícia Valderrama ${ }^{\oplus *, b}$ and \\ Eduardo Carasek ${ }^{\circ *, a}$ \\ ${ }^{a}$ Universidade Federal de Santa Catarina (UFSC), P.O. Box 476, 88040-900 Florianópolis-SC, Brazil \\ ${ }^{b}$ Universidade Tecnológica Federal do Paraná(UTFPR), 87301-899 Campo Mourão-PR, Brazil
}

\begin{abstract}
A potential eco-friendly method without organic solvents is presented by integrating a chromatographic fingerprint and multivariate control chart based on Q residuals to differentiate grape juices from different farming practices. The sample preparation was only water dilution, and the mobile phase was water acidified with sulfuric acid, which can be readily neutralized before its disposal. The proposed method is shown to be a simple way to distinguish between organic and non-organic grape juices in a non-target way, successfully evaluating an external validation data set, where organic and non-organic samples were correctly assigned. Through the chromatographic profile, it is possible to suggest that one of the species responsible for this distinction may be from the anthocyanins class.
\end{abstract}

Keywords: chemometrics, PCA, Q residuals, chromatography, eco-friendly method

\section{Introduction}

Grapes and their derivatives present health benefits, and their consumption has increased over the years. ${ }^{1}$ With an increased focus on healthy products, the production and consumption of organic foods, obtained from specific farming practices, have risen significantly in Brazil (regulated by the Brazilian Law 10.831 of December 23, 2003), ${ }^{2}$ and the production of organic grapes being among the five crops with the largest area of cultivation in the world. ${ }^{3}$

The organic farming practices purpose is to offer healthy products, free from intentional contaminants, preserving the biological diversity of natural ecosystems, with responsible use of water, soil, air, and other natural resources, always considering sustainability and respecting social and cultural relations. ${ }^{2}$ Even more, agriculture is a significant contributor to global greenhouse gas emissions. Organic farming practices might reduce emissions through decreased use of farm inputs and increased soil carbon sequestration. ${ }^{4}$

In line with the consumption increases, benefits provided by grapes such as biological activities,

*e-mail: patriciav@utfpr.edu.br, pativalderrama@gmail.com; eduardo.carasek@ufsc.br

Editor handled this article Emanuel Carrilho (Associated) antioxidant, and anticancer properties, ${ }^{5}$ consequently, increase the demand for quality control of grape and their products. On the other hand, consumers pay an extra price for food products with a declaration of "organic" on the label, so using analytical methodologies that differentiate grape juices from different farmer practices may represent a suitable approach.

Organic grape juices were investigated under antioxidant capacity, physicochemical properties, chemical composition,,$^{3,5-8}$ and anthocyanin content. ${ }^{9}$ Moreover, although the not consensual on the differences in chemical markers and antioxidant activity for grape juice produced from different farming systems, some works pointed that grape juice under different crop systems presented a variation in its chemical compounds. ${ }^{7,8}$ In this scenario, the evaluation of organic foods, produced under-regulated systems, appears as a trend.

The qualitative methods can identify adulterations in products and are currently on the rise mainly due to their screening potential. This strategy is named non-target analysis since the fingerprint of the sample is employed. ${ }^{10}$ Moreover, non-target analysis gaining more space in food quality control, ${ }^{10}$ while the fingerprint can be obtained by different techniques, depending on the availability and interest of the study. 
From the non-target context, molecular spectroscopic such as middle infrared, ${ }^{11,12}$ or ultraviolet-visible (UV-Vis), ${ }^{13}$ have been reported. However, the high-performance liquid chromatography (HPLC) for fingerprint in food matrices has advantages over these other techniques,,$^{14}$ such as bringing significant marker results that get compositional information. Furthermore, by coupling HPLC with chemometrics, food adulteration is successfully detected, and the quality can be assessed. ${ }^{14}$

Among chemometric tools for food evaluation, unsupervised or exploratory ones like principal component analysis (PCA) are usually employed in food industries for quality and process monitoring..$^{15}$ From this unsupervised tool, it is possible to implement a criterion for supervision, for example, by using $\mathrm{Q}$ residuals.

In this context, this work aims to distinguish grape juice from different farming systems by integrating HPLC fingerprinting and multivariate control charts in a non-target analysis. Nonetheless, food samples from organic and conventional farming practices have been investigated using several analytical techniques, such as proton nuclear magnetic resonance $\left({ }^{1} \mathrm{H} \mathrm{NMR}\right),{ }^{16,17}$ isotope ratio mass spectrometry (IRMS), ${ }^{17-19}$ near-infrared (NIR) spectroscopy, ${ }^{20,21}$ and UV-Vis spectroscopy. ${ }^{22}$

Molecular spectroscopy such as NIR and UV-Vis is simpler but has a low resolution and lacks selectivity. An advantage of ${ }^{1} \mathrm{H}$ NMR refers to all ${ }^{1} \mathrm{H}$ nucleus in a sample have the same sensitivity, then for this spectroscopy, the reproducibility is high. On the other hand, the ${ }^{1} \mathrm{H}$ NMR spectroscopic dispersion is limited (about $12 \mathrm{ppm}$ ). This fact will imply overlapped signals (especially with complex samples). Furthermore, the signals for compounds in a lower concentration are often weak and unclear, even if those signals do not overlap with other ones. ${ }^{23}$

Concerning IRMS, the most significant property in this technique for differentiating organic and non-organic foods is the ${ }^{15} \mathrm{~N} /{ }^{14} \mathrm{~N}$ ratio, resulting from the recycling of organic material as fertilizer. However, two processes mimic recycling fertilization with manure and, in the opposite direction, the cultivation of nitrogen-fixing plants such as legumes. ${ }^{24}$ In this scenario, HPLC analysis, a versatile technique with high selectivity, allows establishing a fingerprint for samples. A promising method for food identification produced under different farming practices can be proposed when coupling HPLC fingerprint and chemometrics. Thus, this work shows an application for organic and non-organic grape juice evaluation made by HPLC and chemometrics in an ecofriendly way. The acidified water is employed rather than organic solvents as the mobile phase. So, it meets a green method that claims to reduce or not to use solvents and which the mobile phase can be neutralized before its disposal.

\section{Experimental}

\author{
Samples
}

A total of 33 commercial samples of grape juice from the Rio Grande do Sul, Brazil, were analyzed, being 11 different brands (5 organic brands and 6 non-organic brands). Three bottles of different batches were purchased for each brand, totaling 33 bottles (15 organic and 18 non-organic samples). An aliquot of $500 \mu \mathrm{L}$ of grape juice was diluted in $1.0 \mathrm{~mL}$ of ultrapure water. ${ }^{25}$

\section{Equipment and analytical procedure}

The analysis was performed using an HPLC with a diode array detector (DAD) system (Dionex Ultimate 3000 HPLC, Thermo Scientific, San Jose, CA, USA). The chromatographic conditions were defined according to Coelho et al. ${ }^{25}$ with some modifications. The main ones are regarding the separation mechanism (i.e., column type) and detection wavelength. An aliquot of $500 \mu \mathrm{L}$ of grape juice was diluted in $1.0 \mathrm{~mL}$ of ultrapure water, and $10 \mu \mathrm{L}$ was injected without replicates. The $\mathrm{C} 18$ column was Hi-Plex H $(300 \times 7.7 \mathrm{~mm})$ (Agilent, Santa Clara, CA, USA) with internal particles of $8.0 \mu \mathrm{m}$. The column compartment temperature was maintained at $70^{\circ} \mathrm{C}$. The flow rate applied was $0.5 \mathrm{~mL} \mathrm{~min}^{-1}$ with a run time of $17 \mathrm{~min}$. The mobile phase was $4.0 \mathrm{mmol} \mathrm{L}^{-1} \mathrm{H}_{2} \mathrm{SO}_{4}$ (Synth, Diadema, Brazil) in ultrapure water. The detection was carried out at $240 \mathrm{~nm}$.

\section{Chemometrics}

The chromatograms were aligned through the icoshift algorithm in the Matlab software version R2007b (The Mathworks Inc., Natick, MA, USA). ${ }^{26}$ This algorithm is based on an insertion/deletion model to shift intervals of chromatograms. It relies on an efficient fast Fourier transform, and additional information regarding the mathematical steps can be found in Tomasi et al. ${ }^{27}$ Furthermore, the code for the icoshift algorithm is free available for download. ${ }^{28}$

The aligned chromatograms were employed for multivariate control charts development. An external validation data set was used in this study to check the performance of the multivariate control charts based on $\mathrm{Q}$ residuals. Organic samples were randomly splitting, and $2 / 3$ were used in a training set and $1 / 3$ used as the external validation set. Then, the organic samples in the external validation set were not used for the training step.

Multivariate control charts are the plots of distinct parameters, such as Q residuals from PCA analysis. The 
$Q$ residuals are a lack-of-fit statistic that can be used to indicate how well the pre-established model is describing the sample, i.e., the $\mathrm{Q}$ statistic shows how well each sample conforms to the model. It measures the difference, or residual, between a sample and its projection into the $\mathrm{k}$ factors retained in the model. Equation 1 shows how this parameter is estimated for the $\mathrm{i}^{\text {th }}$ sample in $\mathbf{X}\left(\mathbf{x}_{\mathrm{i}}\right)$ as the sum of squares of each sample (row) of the residual matrix $(\mathbf{E}):^{15}$

$\mathrm{Q}_{\mathrm{i}}=\mathbf{e}_{\mathrm{i}} \mathbf{e}_{\mathrm{i}}^{\mathrm{T}}=\mathbf{x}_{\mathrm{i}}\left(\mathbf{I}-\mathbf{P}_{\mathrm{k}} \mathbf{P}_{\mathrm{k}}^{\mathrm{T}}\right) \mathbf{x}_{\mathrm{i}}^{\mathrm{T}}$

where $\mathbf{e}_{\mathrm{i}}$ is the $\mathrm{i}^{\text {th }}$ row of $\mathbf{E}, \mathbf{I}$ is the identity matrix of appropriate size ( $n$ by $n$ ), and $\mathbf{P}_{k}$ is the matrix of the $k$ loadings vectors retained in the PCA model.

\section{Results and Discussion}

Greening the analytical methods is gaining high interest among researchers and these analytical green methods are named also as eco-friendly methods. Because of the monetary and ecological impact of using large amounts of organic solvents and waste disposal, the analytical community is directed to implement the principles of green analytical chemistry in analytical laboratories and substitute polluting analytical methodologies with green ones. Chromatographic techniques can be greener in this scenario at all analysis steps, from sample collection to separation. ${ }^{29}$ In this work, coupling chromatographic fingerprint and chemometrics made it possible to only use dilution for sample preparation. Furthermore, acidified water was used as a mobile phase, and the result was a successful distinction between organic and non-organic grape juices.

The visual inspection of the raw chromatograms (Figure 1a) suggests around six peak clusters comprised of one to two peaks and variability in retention time across samples. Moreover, retention time alignment is crucial as a pre-treatment step before applying multivariate tools, i.e., the same underlying process must be associated with the same variables in all the samples. When this does not occur, more components (factors) are required to explain the additional variation. Thus, for chromatographic applications, the retention time for the same compound must be invariant across samples. Without meeting this condition, the chemical interpretation of the components and/or factors can be compromised. ${ }^{27}$ Due to this, the chromatographic retention time shifts were corrected (Figure 1b) using the icoshift algorithm (four intervals and the mean of chromatograms as a target). An evident correction can be visually observed mainly on peaks for nearly 14 and 16 min by comparing Figures 1a and $1 \mathrm{~b}$. Only 1 min was running after the last eluted peak.
On the other hand, 5 to 7 min usually ensures all the remaining compounds in the column were eluted. However, in this case, the column equilibrium was reached with isocratic elution. Moreover, no peaks are present in the first 4 min run, which justify this procedure.
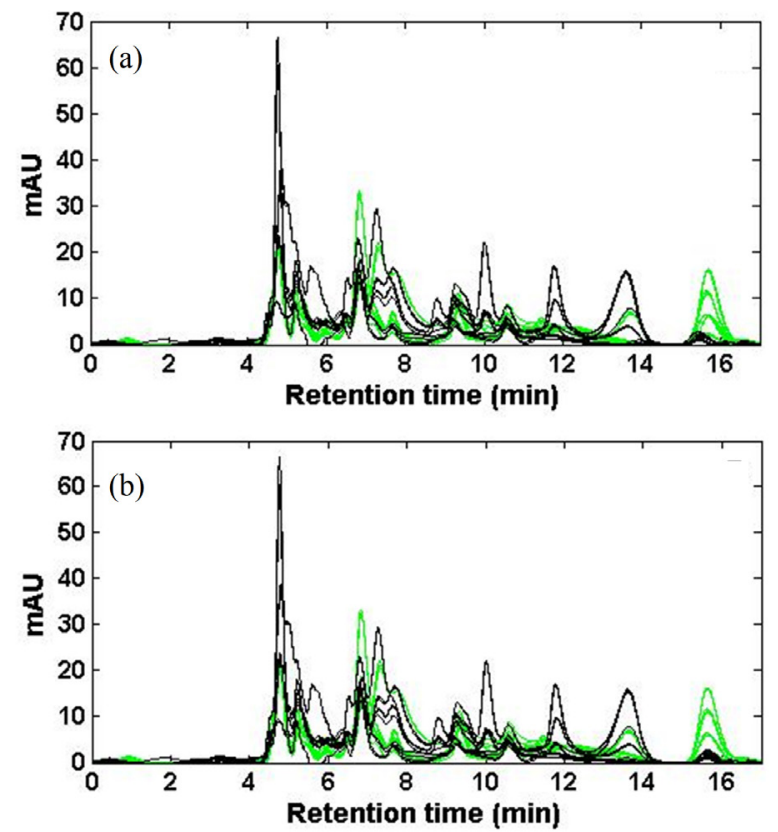

Figure 1. Raw chromatogram (a) and chromatogram after alignment (b). (-) Organic samples, (-) non-organic samples.

By direct observation of the aligned chromatograms, it is possible to conclude about organic and non-organic samples on retention time $15.7 \mathrm{~min}$, where it was observed a lower peak for non-organic samples, and a more intense peak for organic ones. However, regarding the chromatogram results until $14 \mathrm{~min}$, it is challenging to conclude concerning organic or non-organic samples. So, a method was proposed here by integrating the chromatographic fingerprint and multivariate control chart based on $\mathrm{Q}$ residuals in a nontarget analysis.

The first step to obtaining multivariate control charts based on Q residuals is to build a PCA model, using only organic samples in the training set. A data set with organic and non-organic samples are employed in the future in the external validation step. PCA was realized with meancentered chromatograms and three principal components (PC), representing $96.61 \%$ of cumulative variance. The results were plotted using $Q$ residuals values against samples index (Figure 2), showing efficient discrimination between organic and non-organic samples with $99 \%$ confidence.

In Figure 2, a clear differentiation can be observed for all brands of non-organic juices in the external validation data set, effectively distinguishing all non-organic samples and evaluating organic ones in the external validation set. 


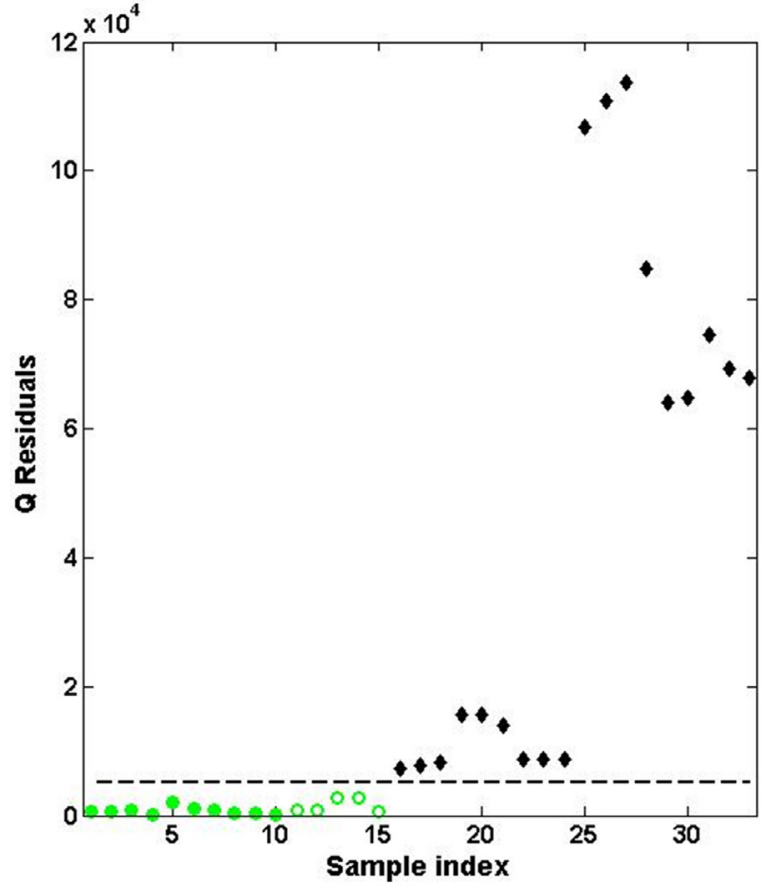

Figure 2. Multivariate control chart results. (๑) Organic samples in training, $(O)$ organic samples in external validation, $(\diamond)$ non-organic samples in external validation.

Control charts based on $\mathrm{Q}$ residuals were successfully reported, for example, from Raman spectroscopy data in the online control of glucose fermentation by Saccharomyces cerevisiae, ${ }^{30}$ from near-infrared (NIR) spectra, ${ }^{15,31}$ and middle (MIR) spectra ${ }^{15}$ to the evaluation of adulterated freeze-dried açai pulp. Here, the results proved that chromatographic fingerprint could be successfully integrated to control chart based on Q residuals, in a nontarget way, to evaluate organic and non-organic grape juice.

Although the primary objective drives a non-target analysis, a discussion regarding the chemical composition possibilities were considered. From the literature, the main and most abundant phenolics present in wines and grape juices belong to the families of flavonols and phenolic acids. ${ }^{6,32-34} \mathrm{In}$ this work, the detection was used at $240 \mathrm{~nm}$ since many peaks were present (fingerprint fulfilled). Moreover, the purpose was fulfilled considering the non-target analysis definition (determinations performed when potential analytes are not limited in their number and origin)..$^{35}$

Nonetheless, Dani et al..$^{7}$ showed that organic grape juices presented statistical differences with higher values for total polyphenols and resveratrol when compared with non-organic grape ones. Polyphenols have a chemical structure derived from benzene, coupled to a hydrophilic group. Considering how the polyphenolic rings attach, they are classified into four families: flavonoids (flavones, flavanones, catechins, and anthocyanins), phenolic acids, lignans, and stilbenes (resveratrol). Furthermore, no differences were observed by Granato et al. ${ }^{6}$ for trans-resveratrol in purple grape juices produced in Brazil under different crop systems. Then, based on those references ${ }^{6,7}$ it can be suggested that the resveratrol was not the compound responsible for differentiating organic and non-organic grape juices.

Regarding the first chromatographic peaks (around 4-6 $\mathrm{min}$ ), they present lower intensities for organic grape juices when compared to non-organic grapes ones. Vian et al. ${ }^{9}$ reached that the total content in anthocyanins during the ripening of the conventionally-grown grapes was significantly higher than that found in the organic production.

Anthocyanins most found in fruits are mainly derived from six anthocyanidins: pelargonidin, cyanidin, delphinidin, peonidin, pethidine, and malvidin. ${ }^{36}$ Although no differences were achieved for the malvidin-3-glucoside, pelargonidin-3-glucoside contents in grape juices produced in Brazil under different farming practices. ${ }^{6}$ Furthermore, to reach the anthocyanidin classes in grape juices, Li et al. ${ }^{37}$ and Wang et al. ${ }^{38}$ employed HPLC with mass spectrometry (MS) detection (electrospray ionization MS/MS detection). Then, a simple and less selective method based on liquid chromatography with detection in only one wavelength when coupled with chemometrics can achieve valuable information concerning grape juice farming practices without a total anthocyanin class identification.

\section{Conclusions}

An eco-friendly chromatographic method was proposed to evaluate grape juice obtained from different farming practices. This greenness method is based on sample preparation by water dilution and mobile phase using acidified water. When coupled with a multivariate control chart based on $\mathrm{Q}$ residuals, the proposed method successfully distinguishes organic and non-organic grape juices.

The proposed method is a simple way to distinguish between organic and non-organic grape juices in a nontarget way. Through the chromatography profile, it is possible to suggest that the distinction maybe be assigned to a compound from the anthocyanins class.

\section{Acknowledgments}

Coordenação de Aperfeiçoamento de Pessoal de Nível Superior (CAPES), Conselho Nacional de Desenvolvimento Científico e Tecnológico (CNPq), and Central Analítica 
Multiusuário da UTFPR-Campo Mourão (CAMulti-CM) were acknowledge. Patrícia Valderrama thanks $\mathrm{CNPq}$ process 306606/2020-8.

\section{Author Contributions}

Leonardo Valderrama was responsible for conceptualization, data curation, software, validation, visualization, and the original draft; Bogdan Demczuk Jr. for data curation, software; Patrícia Valderrama and Eduardo Carasek for conceptualization, funding acquisition, project administration, writing review, and editing.

\section{References}

1. Spinelli, F. R.; Dutra, S. V.; Carnieli, G.; Leonardelli, S.; Drehmer, A. P.; Vanderlinde, R.; Food Control 2016, 69, 1.

2. Ministério da Agricultura e Agropecuária; Lei No. 10.831, de 23 de dezembro de 2003; Dispõe sobre A Agricultura Orgânica e Dá outras Providências; available at http://www.planalto. gov.br/ccivil_03/leis/2003/110.831.htm, accessed in November 2021.

3. Margraf, T.; Santos, É. N. T.; de Andrade, E. F.; van Ruth, S. M.; Granato, D.; Food Res. Int. 2016, 82, 145.

4. Smith, L. G.; Kirk, G. J. D.; Jones, P. J.; Williams, A. G.; Nat. Commun. 2019, 10, 4641.

5. Granato, D.; Carrapeiro, M. M.; Fogliano, V.; van Ruth, S. M.; Trends Food Sci. Technol. 2016, 52, 31.

6. Granato, D.; Koot, A.; Schnitzler, E.; van Ruth, S. M.; J. Food Sci. 2015, 80, C584

7. Dani, C.; Oliboni, L. S.; Vanderlinde, R.; Bonatto, D.; Salvador, M.; Henriques, J. A. P.; Food Chem. Toxicol. 2007, 45, 2574.

8. Vallverdú-Queralt, A.; Medina-Remón, A.; Casals-Ribes, I.; Lamuela-Raventos, R. M.; Food Chem. 2012, 130, 222.

9. Vian, M. A.; Tomao, V.; Coulomb, P. O.; Lacombe, J. M.; Dangles, O.; J. Agric. Food Chem. 2006, 54, 5230.

10. Callao, M. P.; Ruisánchez, I.; Food Control 2018, 86, 283.

11. Moreira, I.; Scarminio, I. S.; Talanta 2013, 107, 416.

12. Rodriguez-Saona, L. E.; Allendorf, M. E.; Annu. Rev. Food Sci. Technol. 2011, 2, 467

13. Marcheafave, G. G.; Pauli, E. D.; Tormena, C. D.; Ortiz, M. C. V.; Almeida, A. G.; Rakocevic, M.; Bruns, R. E.; Scarminio, I. S.; Talanta 2020, 209, 120591.

14. Esteki, M.; Shahsavari, Z.; Simal-Gandara, J.; Food Res. Int. 2019, 122, 303

15. Caramês, E. T. S.; Alamar, P. D.; Pallone, J. A. L.; Food Res. Int. 2019, 123, 704.

16. Hohmann, M.; Christoph, N.; Wachter, H.; Holzgrabe, U.; J. Agric. Food Chem. 2014, 62, 8530.

17. Hohmann, M.; Monakhova, Y.; Erich, S.; Christoph, N.;
Wachter, H.; Holzgrabe, U.; J. Agric. Food Chem. 2015, 63, 9666.

18. Kaffarnik, S.; Schröder, M.; Lehnert, K.; Baars, T.; Vetter, W.; Eur. Food Res. Technol. 2014, 238, 819.

19. Bateman, A. S.; Kelly, S. D.; Woolfe, M.; J. Agric. Food Chem. 2007, 55, 2664

20. Oliveira, V. M. T.; Baqueta, M. R.; Março, P. H.; Valderrama, P.; Anal. Methods 2020, 12, 701.

21. Fernandez, A. S. T.; Bruni, A. R. S.; Oliveira, V. M. T.; Março, P. H.; Valderrama, P.; Quim. Nova 2020, 43, 1500.

22. Wentzell, P. D.; Gonçalves, T. R.; Matsushita, M.; Valderrama, P.; Anal. Chim. Acta 2021, 1174, 338716.

23. Miao, Z.; Jin, M.; Liu, X.; Guo, W.; Jin, X.; Liu, H.; Wang, Y.; Anal. Bioanal. Chem. 2015, 407, 3405.

24. Förstel, H.; Anal. Bioanal. Chem. 2007, 388, 541.

25. Coelho, E. M.; Padilha, C. V. S.; Miskinis, G. A.; de Sá, A. G. B.; Pereira, G. E.; Azevêdo, L. C.; Lima, M. S.; J. Food Compos. Anal. 2018, 66, 160.

26. MATLAB, version R2007b; The MathWorks, Inc.: Natick, MA, USA, 2007.

27. Tomasi, G.; Savorani, F.; Engelsen, S. B.; J. Chromatogr. A 2011, 1218, 7832.

28. Icoshift - An Ultra Rapid and Versatile Tool for the Alignment of Spectral Datasets; http://www.models.life.ku.dk/icoshift, accessed in November 2021.

29. Shaaban, H.; Anal. Bioanal. Chem. 2016, 408, 6929.

30. Ávila, T. C.; Poppi, R. J.; Lunardi, I.; Tizei, P. A. G.; Pereira, G. A. G.; Biotechnol. Prog. 2012, 28, 1598.

31. Lobato, K. B. S.; Alamar, P. D.; Caramês, E. T. S.; Pallone, J. A. L.; J. Food Eng. 2018, 224, 105.

32. Van Leeuw, R.; Kevers, C.; Pincemail, J.; Defraigne, J. O.; Dommes, J.; J. Food Compos. Anal. 2014, 36, 40.

33. Lima, M. S.; Silani, I. S. V.; Toaldo, I. M.; Corrêa, L. C.; Biasoto, A. C. T.; Pereira, G. E.; Bordignon-Luiz, M. T.; Ninow, J. L.; Food Chem. 2014, 161, 94.

34. Padilha, C. V. S.; Miskinis, G. A.; de Souza, M. E. A. O.; Pereira, G. E.; de Oliveira, D.; Bordignon-Luiz, M. T.; Lima, M. S.; Food Chem. 2017, 228, 106.

35. Milman, B. L.; Zhurkovich, I. K.; TrAC, Trends Anal. Chem. 2017, 97, 179.

36. Março, P. H.; Poppi, R. J.; Scarminio, I. S.; Quim. Nova 2008, $31,1218$.

37. Li, Y.; Ma, R.; Xu, Z.; Wang, J.; Chen, T.; Chen, F.; Wang, Z.; J. Sci. Food Agric. 2013, 93, 1404.

38. Wang, H.; Race, E. J.; Shrikhande, A. J.; J. Agric. Food Chem. 2003, 51, 1839.

Submitted: February 25, 2021 Published online: November 17, 2021 